\title{
METABOLIC BALANCES OF SULFUR IN PATIENTS WITH HEPATOLENTICULAR DEGENERATION AND EFFECT OF THE USE OF D-PENICILLAMINE
}

\author{
Horácto M. Canelas * \\ Francisco B. De Jorge ** Waldir A. Tognola ***
}

Though a great deal of knowledge has been achieved in regard to the metabolic disorders of Wilson's disease, many problems are still unsolved.

The metabolic balances of sulfur in two cases of hepatolenticular degeneration submitted to mixed and vegetarian diets ${ }^{1}$ have been studied. The effect of D-penicillamine on the balance was also analyzed.

\section{MATERIAL AND METHODS}

CASE 1: V. M., a 22-year-old woman whose symptoms had started a year earlier; the neurological examination showed the characteristic facies, bilateral Kayser-Fleischer corneal rings, a severe wing-beating tremor in the upper limbs, unsteady gait, slurred speech, and mild rigidity of the upper limbs. The mean blood serum copper content was $29 \mu \mathrm{g} / 100 \mathrm{ml}$, ceruloplasmin level $3 \mathrm{mg} / 100 \mathrm{ml}$ (Houchin method), aminoaciduria $437 \mathrm{mg} / 24 \mathrm{hr}$. The liver biopsy showed a postnecrotic cirrhosis.

CASE 2: J. C. M., a 27-year-old man whose disease started two years before with mental disorder and a severe tremor in the upper limbs. There were bilateral Kayser-Fleischer rings but muscle tone, gait, and speech were normal. The mean blood serum copper was $45 \mu \mathrm{g} / 100 \mathrm{ml}$, ceruloplasmin $0 \mathrm{mg} / 100 \mathrm{ml}$, aminoaciduria $410 \mathrm{mg} / 24 \mathrm{hr}$. The liver biopsy showed fatty infiltration.

The patients were submitted to a vegetarian diet containing $900 \mathrm{mg}$ of sulfur and to a mixed diet containing $1,350 \mathrm{mg}$.

Case 1 was kept in metabolic balance for 42 days, including 20 days on vegetarian diet, 13 days on mixed diet, and 9 days on vegetarian diet again plus $2,000 \mathrm{mg}$ of D-penicillamine (this daily dosis contains $430 \mathrm{mg}$ of total sulfur, which were computed in the balance). Case 2 was kept in metabolic balance for 34 days, including 12 days on vegetarion diet, 13 days on mixed diet, and 9 days on vegetarian diet plus $2,000 \mathrm{mg}$ of D-penicillamine. Each balance was divided in periods from 3 to 6 days.

From the Departments of Neurology (*Associate Professor, ** Instructor) and Medicine (** Chief of Laboratory), University of São Paulo Medical School, São Paulo, Brazil. 


\begin{tabular}{|c|c|c|c|c|c|c|c|c|c|}
\hline \multirow[t]{2}{*}{ Diet } & Period (1964) & \multirow[t]{2}{*}{ Days } & \multirow{2}{*}{$\begin{array}{l}\text { Ingesta } \\
\text { (mg/day) }\end{array}$} & \multicolumn{2}{|c|}{ Excreta } & \multicolumn{3}{|c|}{ Balance } & \multirow{2}{*}{$\begin{array}{l}\text { Blood serum } \\
(\mathrm{mg} / 100 \mathrm{ml})\end{array}$} \\
\hline & & & & Urine & Feces & $\mathrm{mg} / \mathrm{day}$ & $\begin{array}{l}\text { in the } \\
\text { period } \\
(\mathrm{mg})\end{array}$ & mean (mg) & \\
\hline \multirow[t]{5}{*}{ Mixed } & 17 Mar-20 Mar & 4 & 1,226 & 921 & 249 & +56 & $+\quad 224$ & & 1,385 \\
\hline & 21 Mar-23 Mar & 3 & 1,302 & 869 & 258 & +175 & +525 & & - \\
\hline & 24 Mar-26 Mar & 3 & 1,250 & 1,041 & 289 & -80 & -240 & & 1,360 \\
\hline & 27 Mar-29 Mar & 3 & 1,234 & 1,013 & 253 & -32 & 96 & & - \\
\hline & Total & 13 & & & & & +413 & $31.8 \pm 97.3$ & \\
\hline \multirow[t]{6}{*}{ Vegetarian } & 27 Jan-29 Jan & 3 & 846 & 621 & 188 & +37 & +111 & & 1,458 \\
\hline & 30 Jan-02 Feb & 4 & 872 & 600 & 215 & +57 & $+\quad 228$ & & 1,411 \\
\hline & 03 Feb-05 Feb & 3 & 872 & 619 & 213 & +40 & +120 & & 1,562 \\
\hline & 06 Feb-09 Feb & 4 & 860 & 649 & 241 & -30 & $-\quad 120$ & & 1,218 \\
\hline & 10 Feb-15 Feb & 6 & 866 & 666 & 187 & +13 & 78 & & 1,223 \\
\hline & Total & 20 & & & & & $+\quad 417$ & $20.8 \pm 30.6$ & \\
\hline Vegetarian & 22 Apr-24 Apr & 3 & $870+430$ & 1,080 & 212 & +8 & 24 & & 1,730 \\
\hline plus & 25 Apr-27 Apr & 3 & $875+430$ & 1,352 & 279 & $-\mathbf{3 2 6}$ & $-\quad 978$ & & 1,995 \\
\hline \multirow[t]{2}{*}{ D-penicillamine } & 28 Apr-30 Apr & 3 & $844+430$ & 1,719 & 281 & -726 & $-2,178$ & & 2,269 \\
\hline & Total & 9 & & & & & $-3,132$ & & \\
\hline
\end{tabular}

Table 1 - Metabolic balance of sulfur in patient $V . E$. 


\begin{tabular}{|c|c|c|c|c|c|c|c|c|c|}
\hline \multirow[t]{2}{*}{ Diet } & Period (1964) & \multirow[t]{2}{*}{ Days } & \multirow{2}{*}{$\begin{array}{c}\text { Ingesta } \\
\text { (mg/day) }\end{array}$} & \multicolumn{2}{|c|}{ Excreta } & \multicolumn{3}{|c|}{ Balance } & \multirow{2}{*}{$\begin{array}{l}\text { Blood serum } \\
(\mathrm{mg} / 100 \mathrm{ml})\end{array}$} \\
\hline & & & & Urine & Feces & $\mathrm{mg} / \mathrm{day}$ & $\begin{array}{l}\text { in the } \\
\text { period } \\
\text { (mg) }\end{array}$ & mean (mg) & \\
\hline \multirow[t]{5}{*}{ Mixed } & 19 Aug-21 Aug & $\mathbf{3}$ & 1,252 & 901 & 273 & +78 & +234 & & 1,306 \\
\hline & 22 Aug-25 Aug & 4 & 1,252 & 964 & 230 & +58 & $+\quad 232$ & & 1,285 \\
\hline & 26 Aug-28 Aug & 3 & 1,186 & 943 & 170 & +73 & $+\quad 219$ & & 1,225 \\
\hline & 29 Aug-31 Aug & 3 & 1,252 & 1,005 & 205 & +42 & +126 & & 1,345 \\
\hline & Total & 13 & & & & & +811 & $62.4 \pm 14.1$ & \\
\hline \multirow[t]{5}{*}{ Vegetarian } & 14 Oct-16 Oct & 3 & 806 & 456 & 300 & +50 & +150 & & 1,185 \\
\hline & 17 Oct-19 Oct & 3 & 885 & 669 & 179 & +37 & 111 & & 1,225 \\
\hline & 20 Oct-22 Oct & 3 & 864 & 530 & 326 & +8 & 24 & & 1,045 \\
\hline & 23 Oct-25 Oct & 3 & 827 & 521 & 318 & -12 & 36 & & 1,020 \\
\hline & Total & 12 & & & & & $+\quad 249$ & $20.7 \pm 25.3$ & \\
\hline Vegetarian & 07 Nov-09 Nov & $\mathbf{3}$ & $876+430$ & 1,306 & 215 & -215 & -645 & & 1,515 \\
\hline plus & 10 Nov-12 Nov & 3 & $820+430$ & 1,364 & 322 & -436 & $-1,308$ & & 1,707 \\
\hline \multirow[t]{2}{*}{ D-penicillamine } & 13 Nov-15 Nov & 3 & $804+430$ & 1,666 & 385 & -817 & $-2,451$ & & 2,126 \\
\hline & Total & 9 & & & & & $-4,404$ & & \\
\hline
\end{tabular}

Table 2 - Metabolic balance of sulfur in patient J.C.M. 
At the end of each period feces and alimentary residues, which were collected in glass containers kept in a refrigerator, were weighed, mixed and homogenized in a Wharing Bendor. Duplicate samples of each material were then submitted to the analytical method. The ingesta were the difference between the known sulfur content of each type of diet and the concentration in the alimentary residues. Urine was collected daily under the same conditions as feces, and the 24-hour volume determined.

Total sulfur was determined by the turbidimetric method (De Jorge et al. ${ }^{4}$ ) which was detailed for metabolic balance studies elsewhere (Canelas et al. ${ }^{2}$ ).

R E S U L T S

The results are summarized in Tables 1 and 2 .

C O M M E T S

A positive sulfur balance was evidenced in both patients, particularly in J.C.M. under mixed diet, who showed then an average daily sulfur balance of $+62 \mathrm{mg} \pm 14.1$, significantly higher $(t=5.676, \mathrm{P}<0.001)$ than the normal mean $(23.2 \mathrm{mg} \pm 17.3)$ reported by De Jorge and Cintra ${ }^{3}$.

The administration of D-penicillamine induced a markedly negative balance, mostly through the promotion of a progressive increase of the urinary excretion.

In the blood serum the inorganic sulfur contents were always within normal limits (De Jorge et al. ${ }^{5}$ ) except when the patients were submitted to D-penicillamine therapy, in which case the levels showed a progressive rise.

\section{S U M M A R Y}

The metabolic balances of sulfur in two cases of hepatolenticular degeneration were studied. A positive balance was found in both cases; in one patient submitted to mixed diet, the average was significantly higher than the normal mean.

The administration of D-penicillamine promoted a marked increase in the urinary excretion of sulfur, leading to a strongly negative balance.

The blood sulfur contents, which were normal in the control periods, showed a progressive increment after D-penicillamine was started.

\section{RE S U M O}

Balanços metabólicos do enxôfre em pacientes com degeneração hepatolenticular e efeito da administração de D-penicilamina.

Foram estudados os balanços metabólicos do enxôfre em dois pacientes com degeneração hepatolenticular. Em ambos foi comprovado um balanço positivo; em um paciente submetido a dieta geral, a média foi significantemente superior à normal. 
A administração de D-penicilamina promoveu acentuado aumento da excreção urinária do enxôfre, levando a um balanço fortemente negativo.

As concentrações de enxôfre no sôro sangüíneo, que eram normais no periodo de contrôle, sofreram progressivo incremento após a instituição do tratamento com D-penicilamina.

\section{R E F E R E NCES}

1. CANElAS, H. M.; De JORGE, F. B. \& TOGNOLA, W. A. - Metabolic balances of copper in patients with hepatolenticular degeneration submitted to vegetarian and mixed diets. J. Neurol. Neurosurg. Psychiat. 30:371, 1967.

2. CANElAS, H. M.; De JORGE, F. B. \& TOGNOLA, W. A. - Metabolic balances of sulfur in patients with metachromatic leucodystrophy. Arq. Neuro-Psiquiat. (São Paulo) 26:325, 1968.

3. De JORGE,F. B. \& CINTRA, A. B. U. - Metabolismo do enxôfre. Balanços metabólicos em adultos normais sob dieta geral e sob dieta exclusivamente vegetal. Rev. paul. Med. 69:25, 1966.

4. De JORGE, F. B.; SIlvA, A. G. \& CINTRA, A. B. U. - Determinação quantitativa do enxôfre nos materiais biológicos. Rev. bras. Med. 21:491, 1964.

5. De JORGE, F. B.; CANELAS, H. M. \& ZANINI, A. C. - Metabolismo do enxôfre. Valores normais no sôro sangüineo, liquido cefalorraqueano, saliva, urina e fezes. Rev. paul. Med. 65:332, 1964.

Departamento de Neurologia - Faculdade de Medicina da Universidade de São Paulo - Caixa Postal 3461 - Säo Paulo SP - Brasil. 